\title{
THE DETERMINATION OF ANISOTROPIC SURFACE IMPEDANCE IN ELECTROMAGNETIC SCATTERING*
}

\author{
FIORALBA CAKONI ${ }^{\dagger}$ AND PETER MONK M $^{\ddagger}$
}

\begin{abstract}
We consider the inverse scattering problem of determining the anisotropic surface impedance of a bounded obstacle from far field measurements of the electromagnetic scattered field due to incident plane waves. Such an anisotropic boundary condition can arise from surfaces covered with patterns of conducting and insulating patches. We show that the anisotropic impedance is uniquely determined if sufficient data is available, and characterize the non-uniqueness present if a single incoming wave is used. We derive an integral equation for the surface impedance in terms of solutions of a certain interior impedance boundary value problem. These solutions can be reconstructed from far field data using the Herglotz theory underlying the Linear Sampling Method. We complete the paper with preliminary numerical results.
\end{abstract}

Key words. Anisotropic coatings, electromagnetic inverse scattering, mixed boundary conditions, determination of boundary coefficients.

AMS subject classifications. $65 \mathrm{M} 32,35 \mathrm{~J} 25,78 \mathrm{~A} 46$.

1. Introduction. Anisotropic material constants can cause significant issues for inverse scattering algorithms. For example it is impossible to identify general anisotropic permittivity and permeability in Maxwell's equations from far field data $[11,12]$. This is because the far field pattern is invariant to local changes of variables within the scatterer. Such changes of variables result in different anisotropic coefficients and so the coefficients can only be determined up to such changes of variables. Nevertheless the support of the scatterer can still be identified $[12,2]$.

However, once the anisotropy is limited to a lower dimensional manifold, the question arises if uniqueness is still lost. In this paper we shall consider a scattering problem for the time harmonic Maxwells equations with an anisotropic surface impedance.

Anisotropic impedances arise in practice if the surface is covered with a suitable pattern of conducting patches $[14,20]$. At a macroscopic level these patches result in an effective surface impedance that is anisotropic. Interesting questions arise of how to predict this impedance matrix from a description of the conducting patches [21], but we will take an alternative view that we would like to measure the impedance matrix by using remote scattering measurements.

We are thus led to the problem of reconstructing the shape or surface impedance of an object having an unknown anisotropic surface impedance.

A general reference on how anisotropic impedances can be modeled is the book [14]. The utility of such surfaces, and much of the current interest in "High Impedance Surfaces" results from the work of Sievenpiper [23, 22, 21]. We know of very few papers concerning inverse problems for anisotropic surface coefficients. A relevant paper [13] by Holman discusses both non-uniqueness of the general 3D volume problem for light scattering, and also shows that in dimensions greater than 3 a suitable 2 -tensor can be

\footnotetext{
${ }^{*}$ Received November 1, 2010; accepted for publication February 13, 2011. This research was supported in part by the U. S. Air Force Office of Scientific Research under Grant FA9550-08-1-0138.

${ }^{\dagger}$ Department of Mathematical Sciences, University of Delaware, Newark, Delaware 19716, USA (cakoni@math.udel.edu).

$\ddagger$ Department of Mathematical Sciences, University of Delaware, Newark, Delaware 19716, USA (monk@math.udel.edu). The author acknowledges the support of the IMA, University of Minnesota for a visiting position in the fall of 2010 when some of this work was completed.
} 
reconstructed using boundary data measurements. Our work is motivated by results for scalar impedances in [5] and [3].

In this paper we start by describing the model problem in detail (Section 2) and then prove uniqueness of the surface impedance if multistatic far field data is used. We also discuss uniqueness questions using less data, and in particular show that far field data for just one incoming wave determines the impedance up to a scalar function.

To reconstruct the surface impedance we derive an integral equation based on solutions of an associated interior problem. Based on the linear sampling method, we are able to approximate these solutions using suitable solutions of the far field equation that underlies the linear sampling method [6]. For the sake of ease of presentation we assume here that the boundary of the scattering object is known. Of course one could use the linear sampling method to first reconstruct the boundary of the scatterer and then use our integral equation to reconstruct the surface impedance. It is important to notice that the same indicator function that is used to reconstruct the boundary by the linear sampling method, is also used to approximate the interior fields in the integral equation for the impedance function. Hence our method can be seen as an extension of the linear sampling method to allow the reconstruction of the impedance function after obtaining the boundary. In the case when the boundary is a priori known, there are alternative approaches to reconstruct the impedance function by first reconstructing the total field from the far field measurements and then using the boundary condition to reconstruct the impedance function [7, 19].

We are thus lead to an algorithm for reconstructing surface impedance functions, and even can allow a mixed boundary value problem in which parts of the boundary are metallic (perfectly conducting) and other parts are covered with the impedance coating, without using a priori information on the boundary between these parts. We emphasize, that the reconstruction of the impedance function involves only the solution of the (linear and ill-posed) far field equation for a set of sampling points and of the proposed linear integral equation for the impedance function. Our algorithm requires no solution of any boundary value problem. We finish with a preliminary numerical example and some conclusions.

2. Formulation of the problem. Let $D \subset \mathbb{R}^{3}$ be a bounded region with boundary $\Gamma$ such that $D_{e}:=\mathbb{R}^{3} \backslash \bar{D}$ is connected. Each connected component of $D$ is assumed to be a Lipschitz curvilinear polyhedron. Moreover we assume that the boundary $\Gamma=\Gamma_{D} \cup \Pi \cup \Gamma_{I}$ is split in two disjoint parts $\Gamma_{D}$ and $\Gamma_{I}$ having $\Pi$ as their possible common boundary in $\Gamma$ and that each part $\Gamma_{D}$ and $\Gamma_{I}$ can be written as union of a finite number of open $C^{2}$ - faces $\left(\Gamma_{D}^{j}\right)_{j=1, \ldots N_{D}}$ and $\left(\Gamma_{I}^{j}\right)_{j=1, \ldots, N_{I}}$, respectively and $e_{i j}$ denotes the common edge of two adjacent faces $\Gamma^{i}$ and $\Gamma^{j}$. Let $\nu$ denote the unit outward normal defined almost everywhere on $\Gamma$.

The direct scattering problem for the scattering of a time harmonic electromagnetic plane wave by a partially coated perfect conductor $D$ is to find total electric field $E$ and magnetic field $H$ satisfying

$$
\begin{aligned}
& \operatorname{curl} E-i k H=0 \\
& \operatorname{curl} H+i k E=0
\end{aligned}
$$

in $\mathbb{R}^{3} \backslash \bar{D}$. In particular, the total fields $E$ and $H$ are given by

$$
\begin{aligned}
& E=E^{i}+E^{s} \\
& H=H^{i}+H^{s}
\end{aligned}
$$


where $E^{s}, H^{s}$ are the scattered electric and magnetic field, respectively, satisfying the Silver Müller radiation condition

$$
\lim _{r \rightarrow \infty}\left(H^{s} \times x-r E^{s}\right)=0
$$

uniformly in $\hat{x}=x /|x|$ where $r=|x|$ and the incident electric and magnetic field $E^{i}, H^{i}$ are given by

$$
\begin{gathered}
E^{i}(x):=\frac{i}{k} \operatorname{curl} \operatorname{curl} p e^{i k x \cdot d}=i k(d \times p) \times d e^{i k x \cdot d} \\
H^{i}(x):=\operatorname{curl} p e^{i k x \cdot d}=i k d \times p e^{i k x \cdot d}
\end{gathered}
$$

where $k>0$ is the wave number, $d \in \Omega$ is a unit vector giving the direction of propagation and $p$ is the polarization vector, where $\Omega:=\left\{x \in \mathbb{R}^{3}:|x|=1\right\}$ denotes the unit sphere. On the uncoated part $\Gamma_{D}$ of the boundary $\Gamma$ the total electric field satisfies the perfectly conducting boundary condition, i.e.

$$
\nu \times E=0 \quad \text { on } \quad \Gamma_{D}
$$

We consider here the case where the coating of the portion $\Gamma_{I}$ of the boundary $\Gamma$ is made of an anisotropic material and, as a first approximation of the field inside the thin anisotropic coating, we assume that the total electric and magnetic field satisfies the impedance boundary condition on $\Gamma_{I}$, (see [14] and [20])

$$
\nu \times H-\Lambda \cdot[(\nu \times E) \times \nu]=0 \quad \text { on } \quad \Gamma_{I}
$$

where $\Lambda$ is the real valued tensor impedance which is a function of the coating's parameters and the geometry of the coated portion of the boundary $\Gamma_{I}$. Obviously, $\Lambda$ maps a vector tangential to $\Gamma_{I}$ at a point $x \in \Gamma_{I}$ to a vector tangential to $\Gamma_{I}$ at the same point $x \in \Gamma_{I}$. To be more precise, on a smooth face $\Gamma_{I}^{j}$ let $\nu(x)$ be the smooth outward unit normal vector function to $D$ and let $\hat{t}_{1}(x)$ and $\hat{t}_{2}(x)$ be two perpendicular vectors on the tangential plane to $\Gamma_{I}^{j}$ at the point $x$ such that $\hat{t}_{1}, \hat{t}_{2}, \nu$ form a right hand coordinative system with origin at $x$. Hence the matrix $\Lambda(x)$, the local impedance, is given by the following dyadic expression

$$
\Lambda(x)=\left(\lambda_{11}(x) \hat{t}_{1}(x)+\lambda_{12}(x) \hat{t}_{2}(x)\right) \hat{t}_{1}(x)+\left(\lambda_{21}(x) \hat{t}_{1}(x)+\lambda_{22}(x) \hat{t}_{2}(x)\right) \hat{t}_{2}(x) .
$$

Note that, if $\xi(x)=\alpha \hat{t}_{1}(x)+\beta \hat{t}_{2}(x)$ for some $\alpha, \beta \in \mathbb{C}$, then $\Lambda(x) \cdot \xi(x)$ is the tangential vector given by

$$
\Lambda(x) \cdot \xi(x)=\left(\alpha \lambda_{11}(x)+\beta \lambda_{21}(x)\right) \hat{t}_{1}(x)+\left(\alpha \lambda_{12}(x)+\beta \lambda_{22}(x)\right) \hat{t}_{2}(x) .
$$

From physical considerations we assume that $\lambda_{12}(x)=\lambda_{21}(x)$ for all $x \in \Gamma_{I}^{j}, j=$ $1 \cdots N_{I}$ and, in the following, we refer to $\Lambda$ as symmetric tensor. Now, if we express the magnetic fields in terms of corresponding electric fields, the direct scattering problem for a perfect conductor partially coated with a thin anisotropic dielectric material can be written as

$$
\begin{array}{cl}
\operatorname{curlcurl} E^{s}-k^{2} E^{s}=0 & \text { in } \mathbb{R}^{3} \backslash \bar{D} \\
\nu \times E^{s}=f & \text { on } \Gamma_{D} \\
\operatorname{curl} E^{s} \times \nu-i k \Lambda \cdot\left(\nu \times E^{s}\right) \times \nu=h & \text { on } \Gamma_{I} \\
\lim _{r \rightarrow \infty}\left(\operatorname{curl} E^{s} \times x-i k r E^{s}\right)=0 &
\end{array}
$$


where $f:=-\nu \times\left. E^{i}\right|_{\Gamma_{D}}, h:=-\nu \times \operatorname{curl} E^{i}+i k \Lambda \cdot\left(\nu \times E^{i}\right) \times \nu$ and $E^{i}$ is the incident electric plane wave given by (2.6). In the following we assume that the symmetric tensor $\Lambda$ is in $L_{\infty}\left(\Gamma_{I}\right) \cup C\left(\Gamma_{I}^{j}\right)$ for any smooth face $\Gamma_{I}^{j}, j=1 \cdots N_{j}$. Furthermore we assume that $\Lambda$ is uniformly positive definite, i.e. $\overline{\xi(x)}^{\top} \Lambda(x) \cdot \xi(x) \geq \gamma|\xi(x)|^{2}$ where $\gamma>0$ is a positive constant (independent of $x$ ), for every point $x \in \Gamma_{I}^{j}, j=0 \cdots N_{I}$ and every vector $\xi \in \mathbb{C}^{3}$ tangential to $\Gamma_{I}$ at $x$. We remark that it is reasonable to assume that $\gamma$ is independent of $x$. Indeed, if $\xi(x)=\alpha \hat{t}_{1}(x)+\beta \hat{t}_{2}(x)$ for some $\alpha, \beta \in \mathbb{C}$, then

$$
\overline{\xi(x)}^{\top} \Lambda(x) \cdot \xi(x)=|\alpha|^{2} \lambda_{11}(x)+2 \operatorname{Re}(\bar{\alpha} \beta) \lambda_{12}(x)+|\beta|^{2} \lambda_{22}(x)
$$

and the coefficients $\lambda_{i j}(x)$ involve the physical parameters of the coating and the choosen coordinative system on the tangential plane at the point $x$. Roughly speaking, although the impedance changes with direction, it does not become arbitrary small in any point.

For latter use we also formulate the interior mixed boundary problem related to the scattering problem (2.11)-(2.14)

$$
\begin{array}{cl}
\text { curlcurl } E_{0}-k^{2} E_{0}=0 & \text { in } D \\
\nu \times E_{0}=f & \text { on } \Gamma_{D} \\
\operatorname{curl} E_{0} \times \nu-i k \Lambda \cdot\left(\nu \times E_{0}\right) \times \nu=h & \text { on } \Gamma_{I}
\end{array}
$$

In order to investigate mathematically both problems (2.11)-(2.14) and (2.15)(2.17), we need to define the following Sobolev spaces

$$
\begin{aligned}
H(\operatorname{curl}, D) & :=\left\{u \in\left(L^{2}(D)\right)^{3}: \operatorname{curl} u \in\left(L^{2}(D)\right)^{3}\right\} \\
L_{t}^{2}(\Gamma) & :=\left\{u \in\left(L^{2}(\Gamma)\right)^{3}: \nu \cdot u=0 \text { on } \Gamma\right\} \\
L_{t}^{2}\left(\Gamma_{I}\right) & :=\left\{\left.u\right|_{\Gamma_{I}}: u \in L_{t}^{2}(\Gamma)\right\}
\end{aligned}
$$

and introduce the space

$$
X\left(D, \Gamma_{I}\right):=\left\{u \in H(\operatorname{curl}, D): \nu \times\left. u\right|_{\Gamma_{I}} \in L_{t}^{2}\left(\Gamma_{I}\right)\right\},
$$

equipped with the natural norm

$$
\|u\|_{X\left(D, \Gamma_{I}\right)}^{2}=\|u\|_{H(\operatorname{curl}, D)}^{2}+\|\nu \times u\|_{L^{2}\left(\Gamma_{I}\right)}^{2} .
$$

For the exterior domain $D_{e}$ we define the above spaces in the same way for every $D_{e} \cap B_{R}$, with $B_{R}$ a ball of radius $R$, and denote these spaces by $H_{l o c}\left(\operatorname{curl}, D_{e}\right)$ and $X_{l o c}\left(D_{e}, \Gamma_{I}\right)$, respectively. Furthermore we introduce the trace space of $X$ on the complementary part $\Gamma_{D}$ by

$$
Y\left(\Gamma_{D}\right):=\left\{f \in\left(H^{-1 / 2}\left(\Gamma_{D}\right)\right)^{3}: \exists u \in H_{0}\left(\operatorname{curl}, B_{R}\right), \quad \begin{array}{l}
\nu \times\left. u\right|_{\Gamma_{I}} \in L_{t}^{2}\left(\Gamma_{I}\right) \\
\text { and } f=\nu \times\left. u\right|_{\Gamma_{D}}
\end{array}\right\}
$$

where the ball $B_{R}$ contains $D$ and $H_{0}\left(\operatorname{curl}, B_{R}\right)$ is the space of functions $u$ in $H\left(\operatorname{curl}, B_{R}\right)$ satisfying $\nu \times\left. u\right|_{\partial B_{R}}=0$. It is easy to show that $Y\left(\Gamma_{D}\right)$ is a Banach space with respect to the norm

$$
\|f\|_{Y\left(\Gamma_{D}\right)}^{2}:=\inf \left\{\|u\|_{H\left(\operatorname{curl}, B_{R}\right)}^{2}+\|u\|_{L_{t}^{2}\left(\Gamma_{I}\right)}^{2}\right\},
$$


where the infimum is taken over all functions $u \in H_{0}\left(\operatorname{curl}, B_{R}\right)$ such that $\nu \times\left. u\right|_{\Gamma_{I}} \in$ $L_{t}^{2}\left(\Gamma_{I}\right)$ and $f=\nu \times\left. u\right|_{\Gamma_{D}}$. In particular, $Y\left(\Gamma_{D}\right)$ is in fact a Hilbert space (for more details in this trace space see [4]).

Exactly in the same way as in [4] (see also [17]) by using variational techniques, one can show that both the above mixed boundary value problems are well posed and we state the result in the following theorem.

TheOREm 2.1. Suppose $\Gamma_{I} \neq \emptyset$, then given $f \in Y\left(\Gamma_{D}\right)$ and $h \in L_{t}^{2}\left(\Gamma_{I}\right)$ the following hold:

1. There exists a unique solution $E^{s} \in X_{l o c}\left(\mathbb{R}^{3} \backslash \bar{D}, \Gamma_{I}\right)$ of (2.11)-(2.14) satisfying

$$
\left\|E^{s}\right\|_{X\left(D_{e} \cap B_{R}, \Gamma_{I}\right)} \leq C_{1}\left(\|f\|_{Y\left(\Gamma_{D}\right)}+\|h\|_{L_{t}^{2}\left(\Gamma_{I}\right)}\right) .
$$

2. There exists a unique solution $E_{0} \in X\left(D, \Gamma_{I}\right)$ of (2.15)-(2.17) satisfying

$$
\left\|E_{0}\right\|_{X\left(D, \Gamma_{I}\right)} \leq C_{2}\left(\|f\|_{Y\left(\Gamma_{D}\right)}+\|h\|_{L_{t}^{2}\left(\Gamma_{I}\right)}\right) .
$$

Here $C_{1}>0$ and $C_{2}>0$ are constants independent of $f, h$, but $C_{1}$ depends on the radius $R$ of the ball $B_{R}$.

The scattered electric field $E^{s}$ has the asymptotic behavior [10]

$$
E^{s}(x)=\frac{e^{i k|x|}}{|x|}\left\{E_{\infty}(\hat{x}, d, p)+O\left(\frac{1}{|x|}\right)\right\}
$$

as $|x| \rightarrow \infty$, where $E_{\infty}$ is a tangential vector field defined on the unit sphere $\Omega$ and known as the electric far field pattern.

The inverse problem we consider here is to determine the surface tensor impedance $\Lambda$ in $L_{\infty}\left(\Gamma_{I}\right)$ from a knowledge of $E_{\infty}(\hat{x}, d, p)$ for $d \in \Omega_{0}, \hat{x} \in \Omega_{1}$ and two linearly independent polarization perpendicular to each $d$, where $\Omega_{0}$ and $\Omega_{1}$ are open subsets of the unit sphere $\Omega$. In the following investigation we assume, for simplicity, that $E_{\infty}(\hat{x}, d, p)$ is known for all $d, \hat{x} \in \Omega$. All theoretical results obtained here hold true for the case of limited aperture incident and observation directions due to the analyticity of the far field pattern (see Section 3.2 in [1]). We end this sections by introducing the main tools employed in our investigation

The electric far field pattern define the far field operator $F: L_{t}^{2}(\Omega) \rightarrow L_{t}^{2}(\Omega)$ is then defined by

$$
(F g)(\hat{x}):=\int_{\Omega} E_{\infty}(\hat{x}, d, g(d)) d s(d), \quad \hat{x} \in \Omega,
$$

for $g \in L_{t}^{2}(\Omega)$, where $L_{t}^{2}(\Omega)$ is the space of square integrable unit vectors tangential to $\Omega$. Note that by superposition $F g$ is the electric far field pattern of the exterior mixed boundary value problem (2.11)-(2.14) with $E^{i}:=i k E_{g}$ where $E_{g}$ is the electric field of an electromagnetic Herglotz pair with kernel $g$ given by

$$
E_{g}(x)=\int_{\Omega} e^{i k x \cdot d} g(d) d s(d), \quad H_{g}(x)=\frac{1}{i k} \operatorname{curl} E_{g}(x) .
$$


It is easily seen that $E_{g}, H_{g}$ is an entire solution of the Maxwell's equations. We also introduce the electric dipole with polarization $q$ defined by

$$
E_{e}(x, z, q):=\frac{i}{k} \operatorname{curl}_{x} \operatorname{curl}_{x} q \Phi(x, z) \quad H_{e}(x, z, q):=\operatorname{curl}_{x} q \Phi(x, z)
$$

where $\Phi$ is the fundamental solution of Helmholtz equation in $\mathbb{R}^{3}$ defined by

$$
\Phi(x, z):=\frac{1}{4 \pi} \frac{e^{i k|x-z|}}{|x-z|}, \quad x \neq z \quad \text { and } \quad x, z \in \mathbb{R}^{3} .
$$

If $z \in D$ then $E_{e}(x, z, q)$ and $H_{e}(x, z, q)$ satisfy Maxwell's equations in $\mathbb{R}^{3} \backslash \bar{D}$ and the corresponding electric far field pattern $E_{e, \infty}(\hat{x}, z, q)$ is given by

$$
E_{e, \infty}(\hat{x}, z, q)=\frac{i k}{4 \pi}(\hat{x} \times q) \times \hat{x} e^{-i k \hat{x} \cdot z} .
$$

Finally, we recall the far field equation

$$
F g(\hat{x})=E_{e, \infty}(\hat{x}, z, q) \quad z \in D
$$

where $F g$ is given by (2.20). We have the following result (see [4], Theorem 3.2):

TheOREm 2.2. Let $z \in D$. Then for every $\epsilon>0$ there exists an electric Herglotz wave function $E_{g_{z}^{\epsilon}}$ with kernel $g_{z}^{\epsilon} \in L_{t}^{2}(\Omega)$ such that

$$
\left\|\left(F g_{z}^{\epsilon}\right)(\hat{x})-E_{e, \infty}(\hat{x}, z, q)\right\|_{L_{t}^{2}(\Omega)}<\epsilon .
$$

Furthermore, as $\epsilon \rightarrow 0, E_{g_{z}^{\epsilon}}$ converges in the $X\left(D, \Gamma_{I}\right)$-norm to the unique solution $E_{z} \in X\left(D, \Gamma_{I}\right)$ of the interior mixed boundary value problem

$$
\begin{array}{cl}
\operatorname{curlcurl} E_{z}-k^{2} E_{z}=0 & \text { in } D \\
\nu \times\left[E_{z}+E_{e}(\cdot, z, q)\right]=0 & \text { on } \Gamma_{D} \\
\nu \times \operatorname{curl}\left(E_{z}+E_{e}(\cdot, z, q)\right)-i k \Lambda \cdot\left[\nu \times\left(E_{z}+E_{e}(\cdot, z, q)\right)\right] \times \nu=0 & \text { on } \Gamma_{I}
\end{array}
$$

The above theorem is the basis of the linear sampling method for the reconstruction of $D$ which developed [4] for the case of a scalar valued function surface impedance. The results of [4] hold true word by word for the case of tensor surface impedance as well. Note that the linear sampling method provides a reconstruction of $D$ without knowing a priori whether the scatterer is coated or not,and if so what is the extend and the nature of coating. Therefore, although we assume that $D$ is known and consider only the reconstruction of $\Lambda$, this is not a restriction, since a reconstruction of $D$ can always be obtained before hand by using the linear sampling method. (Note also that the data we use here uniquely determines $D[18,6]$.)

3. Uniqueness of $\Lambda$. Here we show that, under the assumptions stated in the previous section, $\Lambda$ in $L_{\infty}\left(\Gamma_{I}\right)$ is uniquely determined from a knowledge of $E_{\infty}(\hat{x}, d, p)$ for $d \in \Omega_{0}, \hat{x} \in \Omega_{1}$ and two linearly independent polarization perpendicular to each $d$, where $\Omega_{0}$ and $\Omega_{1}$ are open subsets of the unit sphere $\Omega$. To this end we prove the following equality.

TheOREM 3.1. Let $E_{g}$ be the electric Herglotz wave function with kernel $g$ defined by (2.21) and let $E_{g}^{s} \in X_{\text {loc }}\left(\mathbb{R}^{3} \backslash \bar{D}, \Gamma_{I}\right)$ be the solution of (2.11)-(2.14) with incident wave $E^{i}$ replaced by $E_{g}$. Then

$$
\int_{\Gamma_{I}}\left(E_{g}^{s}+E_{g}\right)_{\top} \Lambda \cdot\left(\overline{E_{g}^{s}+E_{g}}\right) \top d s=-\|F g\|^{2}-4 \pi \operatorname{Re}(F g, g)
$$


where $u_{\top}:=(\nu \times u) \times \nu, F$ is the far field operator, $(\cdot, \cdot)$ is the inner product over $L^{2}(\Omega)$ and the integral is understood in the $L_{t}^{2}\left(\Gamma_{I}\right)$ sense.

Proof. First it is shown in [9] that if $E_{1}^{s}$ and $E_{2}^{s}$ are two radiating solutions of the Maxwell equation with far field patterns $E_{\infty}^{1}$ and $E_{\infty}^{2}$ then from the vector Green's formula

$$
\int_{\partial D}\left(\nu \times E_{1}^{s} \cdot \operatorname{curl} \overline{E_{2}^{s}}-\nu \times \overline{E_{2}^{s}} \times \operatorname{curl} E_{1}^{s}\right) d s=-2 i k \int_{\Omega} E_{1, \infty} \cdot \overline{E_{2, \infty}} d s .
$$

Furthermore, if $E^{s} \in X_{l o c}\left(\mathbb{R}^{3} \backslash \bar{D}, \Gamma_{I}\right)$ is a radiating solution to the Maxwell's equation with far field pattern $E_{\infty}$ and $H^{s}=\frac{1}{i k} \operatorname{curl} E^{s}$ then (see [10])

$$
E_{\infty}(\hat{x})=\frac{i k}{4 \pi} \hat{x} \times \int_{\partial D}\left(\nu(y) \times E^{s}(y)+\left(\nu(y) \times H^{s}(y)\right) \times \hat{x}\right) e^{-i k \hat{x} \cdot y} d s(y)
$$

and hence if $E_{h}$ is a electric Herglotz wave function with kernel $h \in L_{t}^{2}(\Omega)$, interchanging the order of integration shows that (see Lemma 4.2 of [9])

$$
\int_{\partial D}\left(\nu \times E^{s} \cdot \operatorname{curl} \overline{E_{h}}-\nu \times \overline{E_{h}} \times \operatorname{curl} E^{s}\right) d s=-4 \pi i k \int_{\Omega} E_{\infty} \cdot \bar{h} d s
$$

Now let $E_{g}^{s} \in X_{l o c}\left(\mathbb{R}^{3} \backslash \bar{D}, \Gamma_{I}\right)$ be the solution of (2.11)-(2.14) with incident wave $E^{i}$ replaced by the Herglotz wave function $E_{g}$ with kernel $g$. Then, using the boundary conditions for $E_{g}^{s}+E_{g}$ together with (3.2) and (3.3), and in addition making use of the fact that the tensor surface impedance is symmetric and of real values we have that

$$
\begin{aligned}
& 2 i k \int_{\Gamma_{I}}\left(E_{g}^{s}+E_{g}\right) \top \Lambda \cdot\left(\overline{E_{g}^{s}+E_{g}}\right) \top d s \\
& =\int_{\partial D}\left(\nu \times\left(E_{g}^{s}+E_{g}\right) \cdot \operatorname{curl} \overline{\left(E_{g}^{s}+E_{g}\right)}-\nu \times \overline{\left(E_{g}^{s}+E_{g}\right)} \times \operatorname{curl}\left(E_{g}^{s}+E_{g}\right)\right) d s \\
& =\int_{\partial D}\left(\nu \times E_{g}^{s} \cdot \operatorname{curl} \overline{E_{g}^{s}}-\nu \times \overline{E_{g}^{s}} \times \operatorname{curl} E_{g}^{s}\right) d s \\
& +\int_{\partial D}\left(\nu \times E_{g}^{s} \cdot \operatorname{curl} \overline{E_{g}}-\nu \times \overline{E_{g}} \times \operatorname{curl} E_{g}^{s}\right) d s \\
& +\int_{\partial D}\left(\nu \times E_{g} \cdot \operatorname{curl} \overline{E_{g}^{s}}-\nu \times \overline{E_{g}^{s}} \times \operatorname{curl} E_{g}\right) d s \\
& =-2 i k \int_{\Omega}\left|E_{g, \infty}^{s}\right|^{2} d s-4 \pi i k \int_{\Omega} \bar{g} E_{g, \infty}^{s} d s-4 \pi i k \int_{\Omega} g \overline{E_{g, \infty}^{s}} d s \\
& =-2 i k(F g, F g)-4 \pi i k(F g, g)-4 \pi i k(g, F g),
\end{aligned}
$$

Finally, dividing by $2 i k$ proves the theorem.

THEOREM 3.2. Let $E_{g}$ be the electric Herglotz wave function with kernel $g$ defined by (2.21) and let $E_{g}^{s} \in X_{\text {loc }}\left(\mathbb{R}^{3} \backslash \bar{D}, \Gamma_{I}\right)$ be the solution of (2.11)-(2.14) with incident wave $E^{i}$ replaced by $E_{g}$. Define

$$
\mathcal{B}:=\left\{f \in L_{t}^{2}\left(\Gamma_{I}\right): f=\left(\nu \times\left(E_{g}^{s}+E_{g}\right)\right) \times\left.\nu\right|_{\Gamma_{I}} \text { for all } g \in L_{t}^{2}(\Omega)\right\} .
$$


Then $\mathcal{B}$ is dense in $L_{t}^{2}\left(\Gamma_{I}\right)$.

REMARK 3.1. Our approach to proving the upcoming uniqueness theorem is based on the use of Herglotz wave functions and is influenced by our reconstruction method which also uses Herglotz functions having a density given by the approximate solution of the far field equation. Of course it is possible to modify Theorem 3.2 where instead of scattered fields corresponding to Herglotz wave functions we could use scattered fields corresponding to plane waves for all incident directions. The fundamental idea of the proof, however, remains essentially the same. Furthermore, the current version of the proof of Theorem 3.2 is suitable for the upcoming proof of injectivity of the integral operator involved in the equation for $\Lambda$, as we remark at the end of Section 4.

Proof. Let $u_{\top}:=(\nu \times u) \times \nu$. It suffices to show that if $\varphi \in L_{t}^{2}\left(\Gamma_{I}\right)$ satisfies

$$
\int_{\Gamma_{I}}\left(E_{g}^{s}+E_{g}\right) \top \varphi d s=0
$$

for all $f:=\left(E_{g}^{s}+E_{g}\right)_{\top} \in \mathcal{B}$ then $\varphi=0$. Suppose (3.4) is true for all such $\left(E_{g}^{s}+E_{g}\right)_{\top}$ and let $U \in X_{l o c}\left(\mathbb{R}^{3} \backslash \bar{D}, \Gamma_{I}\right)$ be the unique solution of

$$
\begin{array}{cl}
\operatorname{curl} \operatorname{curl} U-k^{2} U=0 & \text { in } \mathbb{R}^{3} \backslash \bar{D} \\
\nu \times U=0 & \text { on } \Gamma_{D} \\
\nu \times \operatorname{curl} U-i k \Lambda \cdot(\nu \times U) \times \nu=\varphi & \text { on } \Gamma_{I} \\
\lim _{r \rightarrow \infty}(\operatorname{curl} U \times x-i k r U)=0 &
\end{array}
$$

Then since $\left(E_{g}^{s}+E_{g}\right)_{\top}$ on $\Gamma_{D}$, using the boundary conditions and the integral representation formula, we have that

$$
\begin{aligned}
0 & =\int_{\partial D}\left(E_{g}^{s}+E_{g}\right)_{\top} \varphi d s=\int_{\partial D}\left(E_{g}^{s}+E_{g}\right)_{\top}(\nu \times \operatorname{curl} U-i k \Lambda \cdot(\nu \times U) \times \nu) d s \\
& =\int_{\partial D}\left[\left(E_{g}^{s}\right)_{\top}(\nu \times \operatorname{curl} U)-i k\left(E_{g}^{s}\right)_{\top} \Lambda \cdot U_{\top}+\left(E_{g}\right)_{\top}(\nu \times \operatorname{curl} U)-i k\left(E_{g}\right)_{\top} \Lambda \cdot U_{\top}\right] d s
\end{aligned}
$$

Since the matrix $\Lambda$ is symmetric and the boundary condition for $E_{g}^{s}+E_{g}$ we can further continue

$$
\begin{aligned}
0 & =\int_{\partial D}\left[\left(E_{g}^{s}\right)_{\top}(\nu \times \operatorname{curl} U)+U_{\top}\left(-\left(\nu \times \operatorname{curl} E_{g}^{s}\right)-\left(\nu \times \operatorname{curl} E_{g}\right)+i k \Lambda \cdot\left(E_{g}\right)_{\top}\right)\right] d s \\
& +\int_{\partial D}\left[\left(E_{g}\right)_{\top}(\nu \times \operatorname{curl} U)-i k\left(E_{g}\right)_{\top} \Lambda \cdot U_{\top}\right] d s \\
& =\int_{\partial D}\left[\left(E_{g}^{s}\right)_{\top}(\nu \times \operatorname{curl} U)-U_{\top}\left(\nu \times \operatorname{curl} E_{g}^{s}\right)\right] d s \\
& +\int_{\partial D}\left[\left(E_{g}\right)_{\top}(\nu \times \operatorname{curl} U)-U_{\top}\left(\nu \times \operatorname{curl} E_{g}\right)\right] d s .
\end{aligned}
$$

Since both $E_{g}^{s}$ and $U$ are radiating solutions of the Maxwell equation we have that the first integral is zero and hence we conclude that

$$
0=\int_{\partial D}\left[\left(E_{g}\right)_{\top}(\nu \times \operatorname{curl} U)-U_{\top}\left(\nu \times \operatorname{curl} E_{g}\right)\right] d s \quad \text { for all } g \in L_{t}^{2}(\Omega) .
$$


This implies that

$$
\frac{4 \pi}{i k} U_{\infty}(d)=d \times \int_{\partial D}[-i k(n \times U)+(\nu \times \operatorname{curl} U) \times d] e^{-i k x \cdot d} d s
$$

whence $U_{\infty}(d)=0$ which implies $U=0$ by Rellich's lemma. Hence $\varphi=0$.

We are now ready to prove the following uniqueness theorem. We assume that $\partial D$ is fixed and let $\Omega_{0}$ and $\Omega_{1}$ be two open subsets (possibly the same) of the unit sphere $\Omega$.

THEOREM 3.3. Let $E_{\infty, n}$ be the far field pattern of $E_{n}^{s}$ where $E_{n}^{s}$ is the solution of (2.11)-(2.14) corresponding to $\Lambda=\Lambda_{n}$, with support $\bar{\Gamma}_{I}=\bar{\Gamma}_{I n}$, for $n=1,2$. If $E_{\infty, 1}(\hat{x}, d, p)=E_{\infty, 2}(\hat{x}, d, p)$ for $d \in \Omega_{0}, \hat{x} \in \Omega_{1}$ and two linearly independent polarization perpendicular to each $d$, then $\Gamma_{I 1}=\Gamma_{I 2}:=\Gamma_{I}$ and $\Lambda_{1}(x)=\Lambda_{2}(x)$ on every smooth face $\Gamma_{I}^{j}, j=1 \ldots N_{j}$.

Remark 3.2. Uniqueness is in the sense of operators. Depending on the choice of tangent vectors in (2.10) we will obtain different matrix representations, but the operator acts on tangential vector fields in a unique way. The matrix representations are connected by the usual change of basis formula for matrices.

Proof. Since $E_{\infty}(\hat{x}, d, p)$ is a real analytic function of $\hat{x}$ and $d$ on $\Omega \times \Omega$ for a fixed polarization $p$ and linear on $p$, the hypothesis of the theorem imply that $E_{\infty, 1}(\hat{x}, d, p)=E_{\infty, 2}(\hat{x}, d, p)$ for $\hat{x}, d \in \Omega$ and $p \in \mathbb{R}^{3}$. For arbitrary fixed $d \in \Omega$ and $p \in \mathbb{R}^{3}$, from Rellich's lemma we have that the corresponding scattered fields $E_{1}^{s}(\cdot, d, p)=E_{2}^{s}(\cdot, d, p)$ coincide in $\mathbb{R}^{3} \backslash \bar{D}$. In particular, by the trace theorem the Cauchy data of $E_{1}^{s}(\cdot, d, p)$ and $E_{2}^{s}(\cdot, d, p)$ coincide on $\partial D$. Hence by Holmgren's theorem and the boundary condition satisfied by $E_{n}^{s}(\cdot, d, p), n=1,2$ we first conclude that $\Gamma_{I 1}=\Gamma_{I 2}:=\Gamma_{I}$ (note that only one incident wave is needed to conclude that the support of the coatings coincide). Next, by linearity we have that $E_{g 1}^{s}=E_{g 2}^{s}$ coincide in $\mathbb{R}^{3} \backslash \bar{D}$ where $E_{g n}^{s}$ is the solution of (2.11)-(2.14) with incident wave $E^{i}$ replaced by the electric Herglotz wave function $E_{g}$ with kernel $g \in L_{t}^{2}(\Omega)$ and $\Lambda=\Lambda_{n}$ supported on the same $\bar{\Gamma}_{I}$, for $n=1,2$. From Theorem 3.1 we have that

$$
\int_{\Gamma_{I}}\left(E_{g}^{s}+E_{g}\right)_{\top}\left[\Lambda_{1}-\Lambda_{2}\right] \cdot\left(\overline{E_{g}^{s}+E_{g}}\right)_{\top} d s=0, \quad \text { for all } g \in L_{t}^{2}(\Omega)
$$

and from Theorem 3.2

$$
\int_{\Gamma_{I}} \xi(x)\left[\Lambda_{1}(x)-\Lambda_{2}(x)\right] \cdot \overline{\xi(x)} d s_{x}=0, \quad \text { for all } \xi \in L_{t}^{2}\left(\Gamma_{I}\right) .
$$

Viewing the symmetric matrix valued function $\Lambda_{1}-\Lambda_{2}$ as a self-adjoint operator on $L_{t}^{2}\left(\Gamma_{I}\right)$ the above equality, and the smoothness of $\Lambda_{1}$ and $\Lambda_{2}$ on each smooth face $\Gamma_{I}^{j}$, implies that $\Lambda_{1}(x)=\Lambda_{2}(x)$ for $x \in \Gamma_{I}^{j}, j=1 \ldots N_{j}$ (c.f. Theorem 9.2-2 of [16]). This proves unqueness for both the support of the coating and the surface impedance.

In the case of a piece-wise smooth scalar impedance function, it is known [8] that the far field pattern corresponding to one fixed $d$ and $p$ uniquely determines the support of the coating as well as the impedance function. In our case of a matrix impedance, one incident wave determines uniquely the support of the coating $\Gamma_{I}$ but 
not the matrix impedance $\Lambda$. This can be seen from the following observation. Let $\Lambda$ be given and satisfy the assumptions stated in Section 2, and let $\Gamma_{I}^{j}$ be one of the smooth coated faces. Let $E^{s}$ be the solution (2.11)-(2.14) corresponding to this $\Lambda$ and the incident plane wave $E^{i}$ for a given incidence direction $d \in \Omega$ and polarization $p \in \mathbb{R}^{3}$ perpendicular to $d$. For an arbitrary but fixed $x \in \Gamma_{I}^{j}$, we denote by $U \in \mathbb{R}^{2}$ the unit vector in the direction of $\left[\nu(x) \times\left(E^{s}(x)+E^{i}(x)\right)\right] \times \nu(x)$ and by $V \in \mathbb{R}^{2}$ a unit vector orthogonal to $U$. Next we construct a positive symmetric matrix valued function $\tilde{\Lambda}$ on $\Gamma_{I}^{j}$ such that for the fixed $x \in \Gamma_{I}^{j}$

$$
\tilde{\Lambda}(x)=(U V)^{\top}\left(\begin{array}{ll}
0 & 0 \\
0 & 1
\end{array}\right)(U V) .
$$

The smoothness of $\Gamma_{I}^{j}$ and the regularity results for $E^{s}$ imply that $\left(\nu \times E^{s}\right) \times \nu$ and $\left(\nu \times E^{i}\right) \times \nu$ is smooth on $\Gamma_{I}^{j}$ and consequently the entries of $\tilde{\Lambda}$ are smooth function on $\Gamma_{I}^{j}$. Note also that from Holmgren's theorem $\left[\nu \times\left(E^{s}+E^{i}\right)\right] \times \nu$ cannot become zero on any open subset of $\Gamma_{I}^{j}$. Now, let $a:=a(x)>0$ be any positive $C\left(\Gamma_{I}^{j}\right)$ function. Then by construction $a \tilde{\Lambda} \cdot\left[\nu \times\left(E^{s}+E^{i}\right)\right] \times \nu=0, a \tilde{\Lambda}$ is not identically zero and $\Lambda+a \tilde{\Lambda}$ satisfies the assumptions stated in Section 2. Hence the impedance condition

$$
\nu \times \operatorname{curl} E^{s}-i k \Lambda \cdot\left(\nu \times E^{s}\right) \times \nu=-\nu \times \operatorname{curl} E^{i}+i k \Lambda \cdot\left(\nu \times E^{i}\right) \times \nu
$$

for $E^{s}$ on $\Gamma_{I}^{j}$ can also be written as

$$
\nu \times \operatorname{curl} E^{s}-i k(\Lambda+a \tilde{\Lambda}) \cdot\left(\nu \times E^{s}\right) \times \nu=\nu \times \operatorname{curl} E^{i}+i k(\Lambda+a \tilde{\Lambda}) \cdot\left(\nu \times E^{i}\right) \times \nu .
$$

Finally the uniqueness for (2.11)-(2.14) with $\Lambda$ replaced by $\Lambda+a \tilde{\Lambda}$ implies that $\Lambda+a \tilde{\Lambda}$ produce the same far field pattern as $\Lambda$.

Conversely, if $\Lambda_{1}$ and $\Lambda_{2}$ give rise to the same far field pattern for a fixed incident plane wave $E^{i}$ then from Rellich's lemma we have that the corresponding scattered fields $E_{1}^{s}$ and $E_{2}^{s}$ coincide, i.e. $E^{s}:=E_{1}^{s}=E_{2}^{s}$ and $\operatorname{curl} E^{s}=\operatorname{curl} E_{1}^{s}=\operatorname{curl} E_{2}^{s}$ outside $D$. In particular their tangential components also coincide on $\Gamma_{I}$. Hence we have

$$
\left(\Lambda_{1}-\Lambda_{2}\right) \cdot\left[\nu \times\left(E^{s}+E^{i}\right)\right] \times \nu=0 \quad \text { on } \quad \Gamma_{I} .
$$

Hence $\Lambda_{1}$ and $\Lambda_{2}$ differs by $a(x) \tilde{\Lambda}$ where $a \in C\left(\Gamma_{I}^{j}\right), j=1 \ldots N_{j}$ and $\tilde{\Lambda}$ is defined as in (3.9).

The above observation shows that one incident plane wave, i.e the far field pattern of the scattered field corresponding to one fixed incident direction $d \in \Omega$ and one polarization $p \perp d$ uniquely determines the support of the coating $\Gamma_{I}$ and determines the matrix impedance up to a scalar function, continuous on any smooth face $\Gamma_{I}^{j}$, $j=1 \ldots N_{j}$.

4. Reconstruction of $\Lambda$. Let $z \in D$ and let $E_{z}$ be the unique solution of (2.26)-(2.28). Define

$$
W_{z}:=E_{z}+E_{e}(\cdot, z, q)
$$

and let $u_{\top}:=(\nu \times u) \times \nu$ be the tangential component of a function $u \in H(\operatorname{curl}, D)$. Note that $\left.\left(W_{z}\right)_{T}\right|_{\Gamma_{I}} \in L_{t}^{2}\left(\Gamma_{I}\right)$ and that $W_{z}$ depends on the artificial polarization $q$ as well. 
Lemma 4.1. For every two points $z_{1}$ and $z_{2}$ in $D$ and polarization $q \in \mathbb{R}^{3}$ we have that

$$
2 k \int_{\Gamma_{I}}\left(W_{z_{1}}\right)_{\top} \Lambda \cdot\left(\bar{W}_{z_{2}}\right)_{\top} d s=-\|q\|^{2} A\left(z_{1}, z_{2}, k, q\right)+k\left(q \cdot E_{z_{1}}\left(z_{2}\right)+q \cdot \bar{E}_{z_{2}}\left(z_{1}\right)\right)
$$

where $W_{z_{1}}, W_{z_{2}}$ are defined by (4.1),

$$
A\left(z_{1}, z_{2}, k, q\right)=\frac{k^{3}}{6 \pi}\left[2 j_{0}\left(k\left|z_{1}-z_{2}\right|\right)+j_{2}\left(k\left|z_{1}-z_{2}\right|\right)\left(3 \cos ^{2} \phi-1\right)\right],
$$

and $\phi$ is the angle between $\left(z_{1}-z_{2}\right)$ and $q$.

Proof. By applying the second vector Green's formula and using the boundary conditions for $E_{z_{1}}$ and $E_{z_{2}}$ on $\Gamma$ we obtain

$$
\begin{aligned}
2 i k & \int_{\Gamma_{I}}\left(W_{z_{1}}\right)_{\top} \Lambda \cdot\left(\bar{W}_{z_{2}}\right) \top d s=\int_{\Gamma}\left(\nu \times W_{z_{1}} \cdot \operatorname{curl} \bar{W}_{z_{2}}-\nu \times \bar{W}_{z_{2}} \cdot \operatorname{curl} W_{z_{1}}\right) d s \\
& =\int_{\Gamma}\left(\nu \times E_{e}\left(\cdot, z_{1}, q\right) \cdot \operatorname{curl} \overline{E_{e}\left(\cdot, z_{2}, q\right)}-\nu \times \overline{E_{e}\left(\cdot, z_{2}, q\right)} \cdot \operatorname{curl} E_{e}\left(\cdot, z_{1}, q\right)\right) d s \\
& +\int_{\Gamma}\left(\nu \times E_{z_{1}} \cdot \operatorname{curl} \overline{E_{e}\left(\cdot, z_{2}, q\right)}-\nu \times \overline{E_{e}\left(\cdot, z_{2}, q\right)} \cdot \operatorname{curl} E_{z_{1}}\right) d s \\
(4.3) & +\int_{\Gamma}\left(\nu \times E_{e}\left(\cdot, z_{1}, q\right) \cdot \operatorname{curl} \bar{E}_{z_{2}}-\nu \times \bar{E}_{z_{2}} \cdot \operatorname{curl} E_{e}\left(\cdot, z_{1}, q\right)\right) d s .
\end{aligned}
$$

One can easily see that if $E \in H(\operatorname{curl}, D)$ and $H=\frac{1}{i k} \operatorname{curl} E$ is a solution of Maxwell's equations and $z \in D$, we have

$$
\begin{aligned}
& \nu \times E_{e}(y, z, q) \cdot \operatorname{curl}_{y} \bar{E}(y)=-\frac{i}{k}(-i k) \operatorname{curl}_{z} \operatorname{curl}_{z} q \Phi(y, z) \cdot(\nu \times \bar{H}(y)) \\
& =-q \cdot \operatorname{curl}_{z} \operatorname{curl}_{z} \Phi(y, z)(\nu \times \bar{H}(y))
\end{aligned}
$$

and

$\nu \times \bar{E}(y) \cdot \operatorname{curl}_{y} E_{e}(y, z, q)=i k \nu \times \bar{E}(y) \cdot H_{e}(y, z, q)=i k q \cdot \operatorname{curl}_{z} \Phi(y, z)(\nu \times \bar{E}(y))$,

and therefore from the Stratton-Chu formula

$$
\int_{\Gamma}\left(\nu \times E_{e}(y, z, q) \cdot \operatorname{curl}_{y} \bar{E}(y)-\nu \times \bar{E}(y) \cdot \operatorname{curl}_{y} E_{e}(y, z, q)\right)=i k q \cdot \bar{E}(z) .
$$

Moreover (see [9]),

$$
\begin{aligned}
\int_{\Gamma}( & \left.\nu \times E_{e}\left(\cdot, z_{1}, q\right) \cdot \operatorname{curl} \overline{E_{e}\left(\cdot, z_{2}, q\right)}-\nu \times \overline{E_{e}\left(\cdot, z_{2}, q\right)} \cdot \operatorname{curl} E_{e}\left(\cdot, z_{1}, q\right)\right) d s \\
& =-2 i k \int_{\Omega} E_{e, \infty}\left(\cdot, z_{1}, q\right) \cdot \overline{E_{e, \infty}\left(\cdot, z_{2}, q\right)} d s \\
& =-\frac{i k^{3}}{8 \pi^{2}} \int_{\Omega}((\hat{x} \times q) \times \hat{x}) \cdot((\hat{x} \times q) \times \hat{x}) e^{-i k \hat{x} \cdot\left(z_{1}-z_{2}\right)} d s \\
& =-\frac{i k^{3}}{8 \pi^{2}} \int_{\Omega}\left(\|q\|^{2}-(\hat{x} \cdot q)^{2}\right) e^{-i k \hat{x} \cdot\left(z_{1}-z_{2}\right)} d s:=-i\|q\|^{2} A\left(z_{1}, z_{2}, k, q\right),
\end{aligned}
$$


where by straightforward calculations

$$
A\left(z_{1}, z_{2}, k, q\right)=\frac{k^{3}}{6 \pi}\left[2 j_{0}\left(k\left|z_{1}-z_{2}\right|\right)+j_{2}\left(k\left|z_{1}-z_{2}\right|\right)\left(3 \cos ^{2} \phi-1\right)\right]
$$

with $j_{0}$ and $j_{2}$ being spherical Bessel functions of order 0 and 2, respectively, and $\phi$ is the angle between $\left(z_{1}-z_{2}\right)$ and $q$. Hence using (4.4) and (4.5) in (4.3) and dividing both sides of (4.3) by $i$ yield the result.

Next we consider a subset $\mathcal{H}$ of $L_{t}^{2}\left(\Gamma_{I}\right)$ defined by

$$
\mathcal{H}:=\left\{\begin{array}{cc}
f=\left.\left(W_{z}\right) T\right|_{\Gamma_{I}} \text { with } W_{z}=E_{z}+E_{e}(\cdot, z, q), \\
\end{array} \quad \begin{array}{c}
f \in \Gamma_{I}, E_{z} \text { the solution of }(2.26)-(2.28) \text { and } q \in \mathbb{R}^{3}
\end{array}\right\},
$$

where $B_{r}$ is a ball of radius $r$ contained in $D$.

Lemma 4.2. $\mathcal{H}$ is complete in $L_{t}^{2}\left(\Gamma_{I}\right)$.

Proof. Let $\varphi \in L_{t}^{2}\left(\Gamma_{I}\right)$ such that for every $z \in B_{r}$

$$
\int_{\Gamma_{I}}\left(W_{z}\right)_{\top} \varphi d s=0
$$

Let $E \in X\left(D, \Gamma_{I}\right)$ be the solution of the interior mixed boundary value problem

$$
\begin{aligned}
& \operatorname{curlcurl} E-k^{2} E=0 \text { in } D, \\
& \nu \times E=0 \text { on } \Gamma_{D}, \\
& \nu \times \operatorname{curl} E+i k \Lambda \cdot E_{\top}=\varphi \text { on } \Gamma_{I} .
\end{aligned}
$$

Then for $z \in B_{r}$ and $q \in \mathbb{R}^{3}$, using the fact that $\left(W_{z}\right)_{\top}=E_{\top}=0$ on $\Gamma_{D}$, the second vector Green's formula, and (4.4), we have that

$$
\begin{aligned}
0 & =\int_{\Gamma_{I}}\left(W_{z}\right) \top \varphi d s=\int_{\Gamma} W_{z}\left(\nu \times \operatorname{curl} E-i k \Lambda \cdot E_{\top}\right) d s \\
& =\int_{\Gamma}\left[E_{z} \cdot(\nu \times \operatorname{curl} E)-i k E_{z} \Lambda \cdot E_{\top}+E_{e}(\cdot, z, q) \cdot(\nu \times \operatorname{curl} E)-i k E_{e}(\cdot, z, q) \Lambda \cdot E_{\top}\right] d s
\end{aligned}
$$

Using the fact that the real matrix $\Lambda$ is symetric we have that $E_{z} \Lambda \cdot E_{\top}=E_{\top} \Lambda \cdot E_{z}$ and $E_{e}(\cdot, z, q) \Lambda \cdot E_{\top}=E_{\top} \Lambda \cdot E_{e}(\cdot, z, q)$ and using the boundary conditions fot $E_{z}$ we can rewrite the above as follows

$$
\begin{aligned}
0 & =\int_{\Gamma}\left[E_{z} \cdot(\nu \times \operatorname{curl} E)-E \cdot\left(\nu \times \operatorname{curl} E_{z}\right)\right] d s \\
& +\int_{\Gamma}\left[-E \cdot\left(\nu \times \operatorname{curl} E_{e}(\cdot, z, q)\right)+i k E_{\top} \Lambda \cdot E_{e}(\cdot, z, q)\right] d s \\
& +\int_{\Gamma}\left[E_{e}(\cdot, z, q) \cdot(\nu \times \operatorname{curl} E)-i k E_{e}(\cdot, z, q) \Lambda \cdot E_{\top}\right] d s \\
& =\int_{\Gamma}\left[E_{e}(\cdot, z, q) \cdot(\nu \times \operatorname{curl} E)-E \cdot\left(\nu \times \operatorname{curl} E_{e}(\cdot, z, q)\right)\right] d s \\
& =-\int_{\Gamma}\left[\left(\nu \times E_{e}(\cdot, z, q)\right) \cdot \operatorname{curl} E-(\nu \times E) \cdot \operatorname{curl} E_{e}(\cdot, z, q)\right] d s=i k q \cdot E(z) .
\end{aligned}
$$


Thus $q \cdot E(z)=0$ holds for all polarizations $q \in \mathbb{R}^{3}$ and $z \in B_{r}$ and hence $E(z)=0$ for $z \in B_{r}$. By the unique continuation principle for the solution of Maxwell's equations in $D$ we now see that $E \equiv 0$ in $D$, whence by the trace theorem $\varphi \equiv 0$ which proves the lemma.

We recall that from Theorem $2.2, E_{z}$ can be approximated by $E_{g_{z}^{\epsilon}}$ where $g_{z}^{\epsilon}$ is the approximate (regularized) solution of the far field equation. Equation (4.2) can now be seen as an integral equation of the first kind for $\Lambda$. Using Lemma 4.2 , it is easy to see in the same way as in the proof of Theorem 3.2 that left hand side of this equation is an injective compact integral operator with positive kernel defined on the subset of positive functions of $L_{\infty}\left(\Gamma_{I}\right)$ onto $L^{2}\left(B_{r}\right)$.

5. Numerical Results. In this section we provide extremely preliminary numerical results. For a given scatterer $D$ (in this case a cube), and given surface impedance $\Lambda$, we can compute the far field pattern using a modification of UWVF code described in [15]. Using this data we can then apply our inversion algorithm to compute an approximation to the surface impedance.

We now summarize the main steps of our algorithm for reconstructing the matrix surface impedance $\Lambda$ provided that the boundary $\partial D$ (or a reconstruction of $\partial D$ by using the linear sampling method) is known. For a fixed $z \in D$ we find $g_{z} \in L^{2}(\Omega)$ by solving the regularized far field equation

$$
F^{*} F g+\alpha I=F^{*} E_{e, \infty}(\cdot, z, q)
$$

where $\alpha$ is the regularization parameter and $F^{*}$ is the $L^{2}$-adjoint of the far field operator. (Note that $g_{z}$ is already available if the linear sampling method is first used to reconstruct $\partial D$ ). Having found $g_{z}$, we then evaluate the corresponding electric field of the Herglotz pair $E_{g_{z}}$. This is done for $M$ points $z$ in $D$. Next for any pair of points $z_{i}, z_{j} \in D, i, j=1 \ldots M$ we set up the integral equation

$$
\begin{aligned}
2 k \int_{\partial D}\left(E_{g_{z_{i}}}+E_{e}\left(\cdot, z_{i}, q\right)\right)_{\top} \Lambda \cdot\left(\bar{E}_{g_{z_{j}}}+\bar{E}_{e}\left(\cdot, z_{j}, q\right)\right)_{\top} d s \\
\quad=-\|q\|^{2} A\left(z_{i}, z_{j}, k, q\right)+k\left(q \cdot E_{g_{z_{i}}}\left(z_{j}\right)+q \cdot \bar{E}_{g_{z_{j}}}\left(z_{i}\right)\right)
\end{aligned}
$$

and then solve this collection of integral equations to obtain an approximation for $\Lambda$.

Our test scatter is the cube $D=[-1 / 2,1 / 2]^{3}$ and $k=7$ with an impedance boundary condition on each face. We have adopted the cube as our example since it is easy to determine tangent vectors on each face, and have chosen a variable scalar surface impedance that is a different constant on each face of the cube as given in Table 1.

We first show a reconstruction of the boundary of the cube using the standard linear sampling method (LSM) as described for example in [6]. Approximately $0.4 \%$ random error is added to the far field pattern, and the LSM is applied using 92 incoming waves and corresponding 92 measurements of the far field pattern. This results in the reconstruction in Fig. 1. Clearly the varying surface anisotropy does not greatly effect the standard LSM surface reconstruction algorithm, although the two-dimensional slice plots of $1 /\|g\|_{L^{2}(\Omega)}$ do show anisotropic effects. We could use this reconstruction to compute surface tangents and hence compute $\Lambda$ but instead we assume that $\Gamma$ is known and now seek only to reconstruct $\Lambda$.

The implementation of our algorithm has two main steps: 

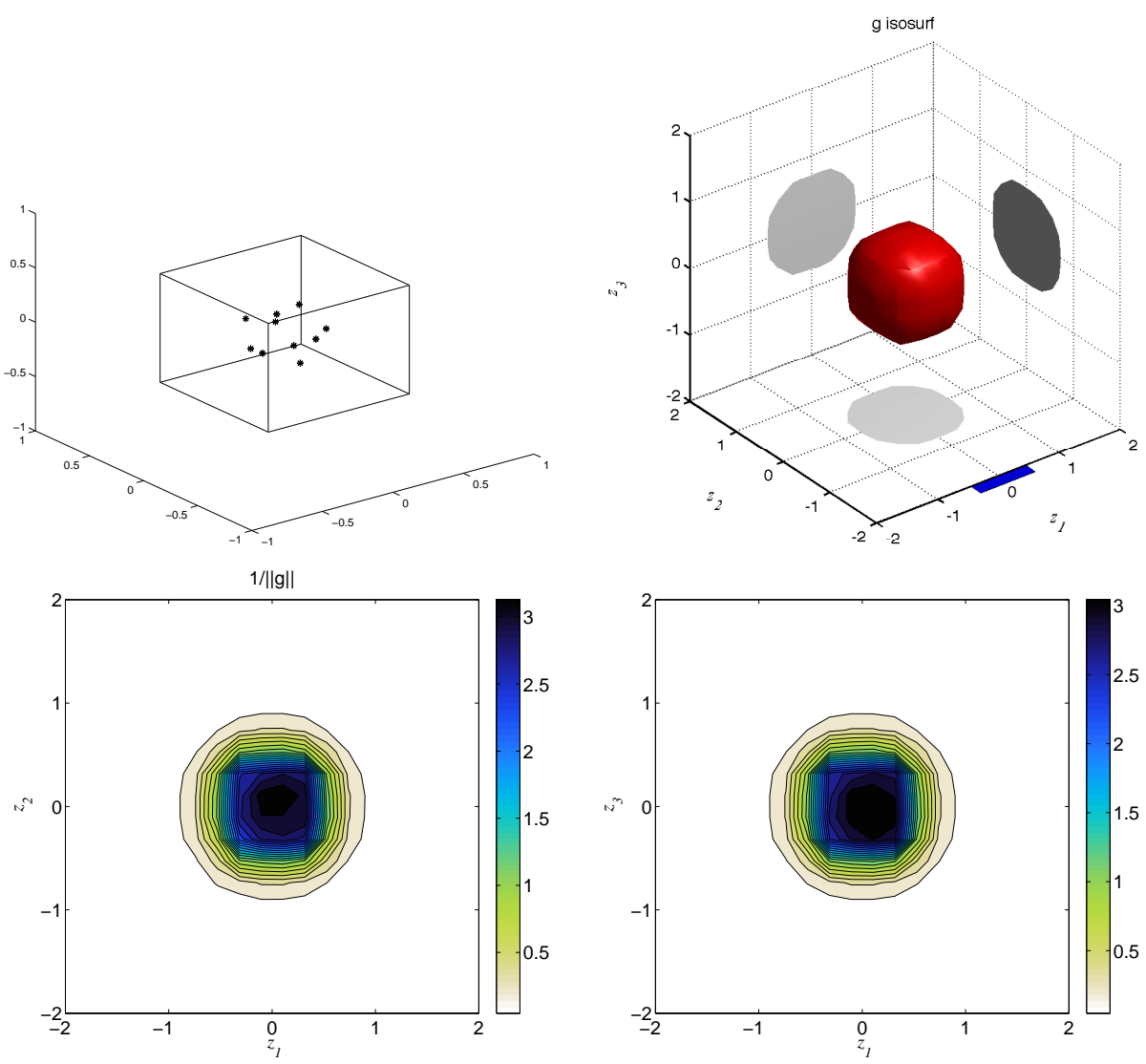

FIG. 1. Reconstruction of the cube scatterer by the standard LSM. Top left: the exact cube showing the position of the auxiliary $z$ points used later to reconstruct $\Lambda$. Top right: iso-surface reconstruction of the cube (the thick line shows the wavelength). The bottom two figures show contour plots of the indicator function on cuts through the search domain. Left: $z_{3}=0$. Right: $z_{2}=0$. Although the reconstruction looks symmetric, we can see patterns in the cross-section plots that arise from using different $\Lambda$ face by face.

1. We choose $M$ points $z_{j} \in D, j=1, \cdots, M$. In our case $M=10$ and the points are chosen at random on the surface of the sphere of radius 0.25 centered at the origin. For each source position $z_{j}$ and for each polarization $q_{\ell}=e_{\ell}, \ell=1,2,3$ where $\left\{e_{\ell}\right\}$ are the usual unit vectors, we can solve (2.24) as usual for the LSM (see [6]). This gives us an approximation to the Herglotz kernels $g_{j, \ell}$ for $1 \leq j \leq M$ and $1 \leq \ell \leq 3$. Evaluating $E_{g}$ in (2.21) using quadrature at the measurement directions gives an approximation for $E_{z}$ in (4.1) for each of the $3 M$ Herglotz kernels.

2. Using the results of the previous step and (4.1) we have an approximation to $W_{z}$ in for $3 M$ choices of $z$ and polarization $q$. Using a piecewise constant representation for $\Omega$ on faces of the scatterer, we can then use (4.2) to compute a $3 M \times 3 M$ matrix problem for the coefficients of $\Lambda$. This requires integration over the surface of $D$, and we take a surface triangulation of $D$ that is sufficiently fine to resolve the incident waves. In fact it is the triangulation used by the forward UWVF - but this is not an inverse crime since the mesh 


\begin{tabular}{||l|c|c|c||}
\hline \hline Face & Exact & Scalar reconstruction & Matrix Reconstruction \\
$y=-.5$ & $\Lambda=2 \mathrm{I}$ & $\Lambda=1.940 I$ & $\Lambda=\left(\begin{array}{cc}1.006 e-01 & -1.650 e-03 \\
-1.650 e-03 & 1.019 e-01 \\
1.945 e+00 & -7.296 e-02 \\
-7.296 e-02 & 1.938 e+00\end{array}\right)$ \\
$z=-.5$ & $\Lambda=1 \mathrm{I}$ & $\Lambda=0.9820 I$ & $\Lambda=\left(\begin{array}{cc}9.830 e-01 & -3.040 e-02 \\
-3.040 e-02 & 9.794 e-01\end{array}\right)$ \\
$z=.5$ & $\Lambda=.3 \mathrm{I}$ & $\Lambda=0.2970 I$ & $\Lambda=\left(\begin{array}{cc}2.958 e-01 & 8.383 e-04 \\
8.383 e-04 & 3.003 e-01\end{array}\right)$ \\
$y=.5$ & $\Lambda=.4 \mathrm{I}$ & $\Lambda=0.3989 I$ & $\Lambda=\left(\begin{array}{cc}4.030 e-01 & -1.261 e-02 \\
-1.261 e-02 & 3.957 e-01\end{array}\right)$ \\
$x=.5$ & $\Lambda=.7 \mathrm{I}$ & $\Lambda=0.6894 I$ & $\Lambda=\left(\begin{array}{cc}6.851 e-01 & 9.804 e-03 \\
9.804 e-03 & 6.897 e-01\end{array}\right)$ \\
\hline \hline
\end{tabular}

TABLE 1

The boundary impedance and its reconstruction. We show results assuming a priori that all boundary impedances are scalar (column 3), and results without any priori assumptions about the boundary impedance (column 4).

\begin{tabular}{|c|c|c|c|}
\hline Face & Exact $\Lambda$ & \multicolumn{2}{|c|}{ Matrix Reconstruction } \\
\hline 5 & $.1 \quad .05$ & 0.1026 & 0.01121 \\
\hline$x=-.5$ & .05 & 0.01121 & 0.1030 \\
\hline & $2 \quad .03$ & 1.682 & -0.05898 \\
\hline$y=-.5$ & $.03 \quad 1.5$ & -0.05898 & 1.587 \\
\hline & $\left(\begin{array}{ll}1 & 0\end{array}\right)$ & 1.148 & -0.05123 \\
\hline$z=-.5$ & $\left(\begin{array}{ll}0 & 1\end{array}\right.$ & -0.05123 & 1.231 \\
\hline$z=5$ & $\left(\begin{array}{ll}.3 & 0\end{array}\right.$ & 0.3028 & 0.005399 \\
\hline & $\begin{array}{ll}0 & .3\end{array}$ & 0.005399 & 0.2994 \\
\hline$u=5$ & $.4 \quad 0$ & 0.3986 & -0.006338 \\
\hline & $\begin{array}{ll}0 & .4\end{array}$ & -0.006338 & 0.3942 \\
\hline$x=5$ & $\left(\begin{array}{ll}.7 & .4\end{array}\right.$ & $(0.5933$ & $0.02344)$ \\
\hline & .7 & 0.02344 & 0.5974 \\
\hline
\end{tabular}

TABLE 2

The boundary impedance and its reconstruction using a mixture of scalar and tensor impedances. The results are less accurate than in Table 1 but provided the off diagonal impedance is large enough, some reconstruction is possible.

is only used to compute integrals on each face. This requires regularization

for large numbers of unknowns (we use Tikhonov regularization).

For the example using a unit cube as scatter, we assume a priori that the tensor $\Lambda$ is constant on each face. Results are shown in Table 1. In the third column we show the results of the algorithm in which we a priori assume that the coefficient $\Lambda=a I$ is piecewise constant on each face. In the fourth column we show the results, still using data from a scalar impedance, but where we have imposed no assumptions on $\Lambda$ Now a matrix is reconstructed on each face, and in each case it is reconstructed to be approximately diagonal. For this small number of unknown coefficients, no regularization of the inversion of (4.2) is needed.

In Table 2 we show results of reconstructing scalar and tensor $\Lambda$. 
6. Conclusion. We have provided a uniqueness result and a reconstruction algorithm for tensor boundary impedances. Unfortunately our numerical results are far from satisfactory since we have only been able to test the algorithm in a few cases. More numerical tests on more general objects and with full tensor impedance would be desirable.

\section{REFERENCES}

[1] F. Cakoni and D. Colton, Combined far field operators in electromagnetic inverse scattering theory, Math. Meth. Appl. Sci., 26 (2003), pp. 413-29.

$[2]-$, A uniqueness theorem for an inverse electromagnetic scattering problem in inhomogeneous anisotropic media, Proc. Edinburgh Math. Soc., 46 (2003), pp. 293-314.

[3] - The determination of the surface impedance of a partially coated obstacle from far field data, SIAM J. Appl. Math., 64 (2004), pp. 709-23.

[4] F. CAKoni, D. Colton, And P. Monk, The electromagnetic inverse scattering problem for partly coated Lipschitz domains, Proc. Royal Soc. Edinburgh, 134A (2004), pp. 661-82.

[5] - The determination of boundary coefficients from far field measurements, Jour. Integral Equations, 22 (2010), pp. 167-191.

[6] - The Linear Sampling Method in Inverse Electromagnetic Scattering, vol. 80 of CBMS, SIAM, Philadelphia, 2011.

[7] F. CAKoni, R. KRess, And C. Schuft, Integral equations for shape and impedance reconstruction in corrosion detection, Inv. Prob., 26 (2010), 095012, 24pp.

[8] D. Colton and R. Kress, Integral Equation Methods in Scattering Theory, Wiley, New York, 1983.

[9] _ Eigenvalues of the far field operator and inverse scattering theory, SIAM J. Math. Anal., 26 (1995), pp. 601-15.

[10] _ Inverse Acoustic and Electromagnetic Scattering Theory, Springer-Verlag, New York, 2nd ed., 1998.

[11] F. Gylys-Colwell, An inverse problem for the Helmholtz equation, Inv. Prob., 12 (1996), pp. 139-56.

[12] P. HÄHNER, On the uniqueness of the shape of a penetrable, anisotropic obstacle, J. Comput. Appl. Math., 116 (2000), pp. 167-180.

[13] S. Holman, Recovering a tensor impedance on the boundary from polarization and phase measurements, Inv. Prob., 25 (2009), 035009 (11pp).

[14] D. Hoppe and Y. Rahmat-Sami, Impedance Boundary Conditions in Electromagnetics, Taylor\& Francis, 1995.

[15] T. Huttunen, M. Malinen, And P. Monk, Solving Maxwell's equations using the Ultra Weak Variational Formulation, J. Comput. Phys., 223 (2007), pp. 731-58.

[16] E. Kreyszig, Introductory Functional Analysis with Applications, Wiley, New York, 1989.

[17] P. Monk, Finite Element Methods for Maxwell's Equations, Oxford University Press, Oxford, 2003.

[18] R. Pike And P. SABatier, eds., Electromagnetic waves scattering: Scattering by obstacles, London, 2001, Academic Press, pp. 191-210.

[19] W. Rundell, Recovering an obstacle and its impedance from Cauchy data, Inv. Prob., 24 (2008), 045003, 22 pp.

[20] T. Senior and J. Volakis, Approximate Boundary Conditions in Electromagnetics, vol. 41 of Approximate Boundary Conditions in Electromagnetics, The Insititution of Electrical Engineers, London, 1995.

[21] D. Sievenpiper, Artificial impedance surfaces for antennas, in Modern Antenna Handbook, C. Balanis, ed., Wiley, 2008.

[22] D. Sievenpiper, J. Colburn, B. Fong, J. Ottusch, and J. Visher, Holographic artificial impedance surfaces for conformal antennas, in Antenna Applications Symposium Digest, Monticello, Illinois, September 2007.

[23] D. Sievenpiper, L. Zhang, R. Broas, N. Alexopolous, and E. Yablonovitch, Highimpedance electromagnetic surfaces with a forbidden frequency band, IEEE Trans. Microwave Theory Tech., 47 (1999), pp. 2059-74. 